\title{
El dispositivo trans: sobre realidades, complejidades y subversiones al aparato cisheterosexual $^{1}$
}

\author{
Jaime Alonso Caravaca Morera ${ }^{2}$
}

Institución: Universidad Federal de Santa Catarina, Brasil

\section{RESUMEN}

Introducción: En la actualidad el análisis de las relaciones e interacciones entre sexos, cuerpos, géneros y sexualidades se centra en la discusión sobre la despatologización y despsiquiatrización de algunas conductas consideradas como ininteligibles.

Desarrollo: Ante este escenario, el presente artículo objetivó analizar las singularidades, complejidades y realidades del dispositivo trans de acuerdo con la (i)lógica cisheterosexual. Las reflexiones aquí planteadas giran en torno de la noción de dispositivo surgida del pensamiento foucaultiano y revelan el impacto que las redes de intervención de saber y poder (panóptico) psiquiátrico han tenido en la consolidación de la patologización de una de las polisémicas y multidimensionales condiciones humanas, como lo es la transexualidad.

Conclusión: A través de este recorrido sucinto es posible (re)pensar el proceso de patologización del dispositivo trans, entendiéndolo como una posibilidad de resistencia y contestación al imperativo cisheteronormativo y a la medicalización de condiciones diversas y por veces inefables.

Palabras claves (DeCS): Transexualidad. Dispositivo. Subjetividad. Salud Colectiva

\footnotetext{
${ }^{1}$ Fecha de recepción: 31 de agosto del 2016

Fecha de aceptación:20 de noviembre del 2016

${ }^{2}$ Enfermero. Doctorando en Enfermería en el Programa de Post-Grado en Enfermería de la Universidad Federal de Santa Catarina,

Brasil (PEN/UFSC).Correo electrónico: jaimealonso.caravaca@ucr.ac.cr
} 


\title{
The trans device: realities, complexities and subversions related to the cisheterosexual system $^{1}$
}

Jaime Alonso Caravaca Morera ${ }^{2}$

Institution: Federal University of Santa Catarina, Brazil

\begin{abstract}
Introduction: By exploring the cartography of social relationships and interactions between genders, bodies, genders and sexualities in contemporary times, this article is aimed at analyzing the peculiarities, complexities, and realities of the trans dispositive according to the current cis-heterosexual (il)logic.

Development:This paper addresses reflections that revolve around the notion of Foucault's thought of dispositive. The aim is to reveal the impact of the strategies of power-knowledge of the Psychiatric (panopticon) science in the consolidation of the pathologization of one of the polysemic human conditions, here in question the transsexuality.
\end{abstract}

Conclusion: Through this succinct route it is possible (re) to think the process of pathologization of the trans device, understanding it as a possibility of resistance and answer to the imperative cisheteronormativo and the medicalization of diverse and sometimes ineffable conditions.

Key Words: Transexuality. Dispositive. Subjectivity.

\footnotetext{
${ }^{1}$ Date of receipt: August 31, 2016

Date of acceptance: November 20, 2016

${ }^{2}$ Nurse. Doctorate in Nursing in the Post-Graduate Program in Nursing of the Federal University of Santa Catarina, Brazil (PEN / UFSC). Email: jaimealonso.caravaca@ucr.ac.cr
} 


\title{
O dispositivo trans: sobre realidades, complexidades e subversões ao aparato cisheterosexual $^{1}$
}

\author{
Jaime Alonso Caravaca Morera ${ }^{2}$
}

Instituição: Universidade Federal de Santa Catarina

\section{RESUMO}

Introdução: $\mathrm{Na}$ atualidade a análise das relações e interações entre os sexos, corpos, gêneros e sexualidades centra-se na discussão sobre a despatologização e despsiquiatrização de alguns comportamentos considerados ininteligíveis. Diante deste cenário, o presente artigo objetivou analisar as singularidades, complexidades e realidades do dispositivo trans, de acordo com a (i)lógica cisheterosexual.

Desenvolvimento: As reflexões aqui colocadas giram em torno da noção de dispositivo surgida do pensamento foucaultiano e revelam o impacto que as redes de intervenção do saber e poder (panóptico) psiquiátrico tem tido na consolidação da patologização de uma das polissémicas e multidimensionais condições humanas: transexualidade.

Conclusão: Através desta viagem rápida possível (re) pensar o processo de patologização do dispositivo de transporte, entendido como uma possibilidade de resistência e de resposta à cisheteronormativo imperativa e a medicalização de várias condições e às vezes indizível.

Palavras chave: Transexualidade. Dispositivo. Subjetividade. Saúde Coletiva

\footnotetext{
${ }^{1}$ Data de recebimento: 31 de agosto de 2016

Data de aceitação: 20 de novembro de 2016

${ }^{2}$ Enfermeira. Doutora em Enfermagem do Programa de Pós-Graduação em Enfermagem da Universidade Federal de Santa Catarina, Brasil (PEN / UFSC) Email: jaimealonso.caravaca@ucr.ac.cr
} 


\section{Revista Electrónica Enfermería Actual en costa Rica}

\section{INTRODUCCIÓN}

Los sistemas filosóficos tradicionales han establecido una conexión axiomática (y muchas veces hasta rizomática) entre el sujeto y el conocimiento, por tanto, defienden la postura de que la naturaleza del sujeto es la génesis y la fuente de la verdad estructural, razón por la que, efectivamente, pensar, analizar y comprender el dispositivo trans $^{1}$ en el actual contexto necro/biopolítico, implica la necesidad de reflexionar sobre territorios de (auto) enunciación de naturaleza polisémica, multifacética y multidimensional, vinculados a las diversas prácticas y subjetividades.

Claramente, las múltiples formas en las que la ontología trans - vinculada en el ámbito de esta investigación al estudio de la naturaleza de la realidad vivencial y situacional - viene siendo retratada a través de los diversos medios de formación de opinión -sean estos académicos, televisivos, vinculados a las redes sociales o demás escenarios de interacción social- revelan la complejidad de las nuevas formas de visibilidad (formas de ser, estar y vivir en el mundo) y enunciación (formas de expresión y presentación), que subyacen en el aparato social disciplinar ${ }^{1}$.

Si se parte de la premisa de que la transexualidad solamente puede ser comprendida si tomamos en consideración la multiplicidad (casi infinita) de aristas que su dimensión discursiva y fenomenológica hospeda, este artículo decanta por pluralizar su definición y hacer referencia siempre al concepto de transexualidades. Dicha postura, en sí, resalta el carácter intersubjetivo, diverso, heterogéneo y multifacético de vivir, ser y presentar una experiencia que, para algunas personas (incluyendo aquí profesionales de la salud), se asocia a la supuesta incongruencia (explícitamente artificial) entre lo designado por una genitalidad (supuestamente) biológica y una teoría performativa (y visiblemente prescriptiva) social de los roles de género.

Efectivamente, esta postura alternativa (y para algunos, subversiva), revela las restricciones, resistencias, reinvenciones y desestabilizaciones que decreta el modelo de inteligibilidad compulsoria del sistema de sexoscuerpos-géneros-sexualidades-identidades ${ }^{1-3}$.

En esta lógica, al adoptarse una postura de reconocimiento de la pluralidad y multiplicidad trans, se resalta la posibilidad de examinar las polifónicas dimensiones éticas, morales, políticas y estéticas de cada individuo. ${ }^{2}$

\footnotetext{
${ }^{I}$ El término 'trans' (en cursiva) en el ámbito de esta reflexión, hace referencia a las personas que se autoidentifican dentro del espectro de género como transexuales (transhombres, transmujeres). Además, podría incluir personas que se identifican dentro y fuera del sistema normativo binario de género, es decir, de la idea simbólica, restrictiva y normativa que tenemos de "masculino" y "femenino". El uso de este concepto a mi parecer, permite revelar mayor fluidez semántica de modo que posibilita la exclusión de algunas clasificaciones jerárquicas.

2 Pienso en la categoría "individuo" a partir de la definición de "sujeto" desarrollada por Foucault (2012). Hablando sobre su pensamiento, el filósofo dice que el enfoque de sus trabajos no era el fenómeno del poder, sino el de crear una historia de los diferentes mecanismos por los cuales los seres humanos se tornan sujetos. Así, desde el punto de vista foucaultiano, el individuo es entendido como aquella forma de ser y estar en el y los mundos, y que emerge a partir de la relación saber-poder y de la relación consigo mismo.
} 


\section{Revista Electrónica Enfermeria Actual en costa Rica}

Partiendo de esta realidad conjunta, es necesario (re)afirmar los diferentes mecanismos de autoenunciación e interacción interpersonal y colectiva, con el intuito de comprender al dispositivo trans como un conjunto de prácticas racionales e involuntarias, por las cuales los seres humanos determinan -de acuerdo con sus interpretaciones- seguir (o no) un conjunto aleatorio y convencional de reglas, al tiempo que lo leen, significan y transforman, utilizando sus historias de vida y necesidades, como brújula cognitiva.

Destacado desde ya como una condición singular en relación con otras presentaciones comportamentales humanas, el dispositivo trans podría ser ampliamente asumido como un objeto de investigación foucaltiano privilegiado y merecedor de esfuerzos analíticos y de comprensión específica ${ }^{4}$, principalmente porque este arte de (re)existencia, resistencia y desafío de los patrones de género histórica y culturalmente impuestos, puede ser encontrado en la mayoría de reflexiones de Michel Foucault, quien se interesó principalmente en analizar la relación del sujeto y sus formas de producción, a partir de los diferentes mecanismos de objetivación que operaban a través de la relación de saber-poder.

Dadas esas irreductibles particularidades filosóficas foucaultianas y otras posturas intelectuales que han colorido las transexualidades, se admite la inconmensurabilidad de los distintos anclajes ontoepistemológicos (algunos de ellos visiblemente anacrónicos) sobre los cuales se apoyaron diferentes nomenclaturas nosológicas vinculados a esa supuesta peculiaridad individual (¿o social?).

Apoyados en esta complejidad bio-necropolítica tempestiva, es que los caminos de este texto se construyen, con el objetivo de analizar las singularidades, complejidades y realidades del dispositivo trans de acuerdo con la (i)lógica cisheterosexual ${ }^{1}$.

En este proceso analítico-reflexivo se visitó -a través de la postura biopolítica foucaultiana- los procesos de consolidación del dispositivo trans, resaltando las nuevas configuraciones y actualizaciones de las líneas de fuerza (bio/necropoder y otros regímenes discursivos y pragmáticos en la contemporaneidad). Sin embargo, se resalta que el presente ejercicio de reflexión no pretende de forma alguna subestimar diferencias irreductibles fácilmente observables entre otros filósofos, obras, momentos y movimientos que han colaborado en la comprensión o, por lo menos, han hecho un esfuerzo por comprender los procesos de sumisión del dispositivo trans a los diferentes regímenes de (bio/necro) poder.

Como se sabe, las personas trans forman parte de un medio social permeado por diversas artimañas y dinámicas de control que objetivan la uniformización humana y la reducción de la diversidad comportamental. Entre esas dinámicas procesuales, se encuentra la psiquiatrización, medicalización o la patologización de algunas (muchas) condiciones/expresiones multisémicas humanas, tal como lo describe Peter Conrad ${ }^{5}$ en su teoría sobre "The medicalization of society". Ante tal realidad, para analizar estas (no tan) recientes inscripciones cognitivas y

\footnotetext{
${ }^{1}$ Se entiende por cisheteronormatividad al marco sociojurídico, descriptivo y prescriptivo que justifica y resalta que las personas se dividen (irremediablemente) de forma natural en dos sexos y dos géneros diferentes y excluyentes (hombres/mujeres, masculino/femenino) y que poseen papeles de comportamiento específicos (performatividades exclusivas). Además, esta noción defiende la postura de que la heterosexualidad es la única identidad sexual del deseo esperable y excluye la diversidad restante. Consecuentemente, esta noción alinea la plasticidad del sexo biológico (principalmente la genitalidad), la identidad de género, el rol de género y las identidades sexuales del deseo, en una misma ecuación.
} 


\section{Revista Electrónica Enfermeria Actual en costa Rica}

vivenciales, es necesario ahondar en las relaciones de saber y poder que, en todo momento y desde diferentes lugares de exposición, incitan, controlan, desafían y consolidan las infinitas subjetividades ${ }^{6-7}$.

Como esencia de lo anterior, a través de esta reflexión se persigue comprender la manera en que las personas que vivencian experiencias localizadas dentro del espectro trans, leen, interpretan, comprenden, negocian y subvierten las normas asociadas al imperativo medicalizador, al tiempo que denuncian las fracturas y debilidades que integran las representaciones ${ }^{1}$ contemporáneas del sexo, cuerpo, género y sexualidad.

\section{DESARROLLO}

La experiencia trans ha sido conceptualizada (y mismo abordada) por diferentes campos de conocimiento, a través de prácticas discursivas arraigadas en nosologías, etiologías y protocolos principalmente clínicos (médicos), aspecto plasmado en varios presupuestos de Michel Foucault quien, desde la arqueología del saber, intentó distanciarse del análisis del enunciado propositivo del conocimiento científico que normalmente fue definido por la relación neutra y objetiva entre el sujeto y el objeto, lo cual perpetúa la convicción de que el objeto y el sujeto no son unidades universales y complementarias, pues ellos se tornan así, únicamente a través de prácticas sociales, teóricas o científicas ${ }^{8}$.

En sentido análogo, para este filósofo los dispositivos son un instrumento formado por un conjunto de prácticas discursivas y no discursivas que poseen una función estratégica de dominación: el poder disciplinar9 Complementariamente, el poder disciplinar obtiene su eficacia entre los discursos teóricos y las prácticas reguladoras.

En términos similares, los dispositivos son una carga de reglas, normas, reglamentos, leyes, postulados, proposiciones morales, éticas, estéticas, rituales e instituciones (dentro de las cuales podemos encontrar a las familias, iglesias, escuelas y prisiones) impuestas a los individuos por medio de un poder externo, pero que se interiorizan en los sistemas de creencias y sentimientos ${ }^{10}$; es decir, son formas de control y resistencia cuya heterogeneidad es caracterizada por la capacidad de unir puntos, de establecer conexiones, de formar redes y de configurar una racionalidad a un determinado campo, sea el material, expresivo, constructivo y/o discursivo ${ }^{3}$.

Articulados de formas y proporciones variadas, tanto en el sentido común como en el foucaultiano, esos dispositivos parecen referirse a la disposición de una serie de prácticas y mecanismos -lingüísticos, no linguísticos, jurídicos y técnicos- que surgen con el objetivo de enfrentar una situación (o condición) específica y causar un efecto.

Su notable recurrencia compite para sedimentar y perpetuar la imagen de un profundo saber y poder que divide las sociedades por medio de conductas que son tomadas como modeladoras en la contemporaneidad. Importa observar de forma más clara y en la perspectiva foucaultiana, que los dispositivos se articulan y se producen en determinados momentos históricos con la función estratégica de producir y sustentar verdades: así

\footnotetext{
${ }^{I}$ Entiendo a las representaciones del sexo, cuerpo, género y sexualidad como símbolos que, como todo símbolo, son discusivos, descriptivos, prescriptivos, unitarios, autoritarios e impositivos.
} 


\section{Revista Electrónica Enfermeria Actual en costa Rica}

aconteció con la sexualidad, a partir de los siglos XVIII y XIX y simultáneamente con la transexualidad, cuya génesis y consolidación se remontan al siglo XIX. Así, la construcción del dispositivo de la transexualidad debe ser entendida dentro de un contexto de medicalización, tanto del cuerpo individual como del cuerpo colectivo ${ }^{9,11-}$ 13

A partir de las discusiones generadas por Michel Foucault ${ }^{12}$, y como se ha explicado anteriormente, el dispositivo trans se configura como un conjunto de experiencias, tesis, postulados y prácticas que se encierran en un régimen de enunciados (erróneamente) patologizadores, que consolidaron la experiencia trans como un trastorno de identidad de género (según el Manual Diagnóstico y Estadístico de los Trastornos Mentales IV $^{14}$ ) y/o como su versión contemporánea de disforia de género (según el $\mathrm{V}^{15}$ ).

En esta línea de raciocinio, la idea de la transexualidad como dispositivo denuncia un conjunto de saberes que, a través de relaciones y prácticas de poder, establecieron toda una organización conceptual y práctica sobre los sexos, los cuerpos, los géneros y las sexualidades, la cual permitió y legitimó esta condición, como un fenómeno médico y jurídico por excelencia, pero particularmente psiquiátrico.

La genealogía de este proceso encuentra resonancia en el dispositivo de la sexualidad y en la fuerza de la scientia sexualis a partir de los ya mencionados siglos XVIII y XIX ${ }^{3,13}$. En sí, todo este proceso de contextualización invita a pensar y reflexionar sobre la forma en que los dispositivos influencian y operan sobre otros dispositivos ${ }^{7}$. Tales dispositivos de control en el ámbito de salud refuerzan la noción de la cisheteronormatividad y producen subjetividades, al tiempo que establecen modelos que refuerzan la idea de lo que significa ser una persona trans, como si la noción ontológica de ser persona ya no representase suficiente complejidad para no protocolar o caracterizar patrones cognitivos de comportamiento.

En esta (des)armonía se funda el pensamiento de que los discursos y prácticas medicalizadoras, pero principalmente psiquiatrizantes y moralizadoras, actúan no solamente en contextos de salud, sino en contextos sociales amplios. Simultáneamente nos alertan de la necesidad urgente de (re)pensar y (re)definir los imperativos normativos que omiten las singularidades en las experiencias trans.

En sentido análogo, cabe señalar que el concepto de dispositivo foucaultiano ofrece una herramienta conceptual y práctica importante para pensar en los procesos de control bionecropolíticos contemporáneos. Efectivamente, sus estudios relacionados con la influencia de la soberanía y el biopoder, la consolidación de las sociedades disciplinares y, consecuentemente, de la biopolítica, revelaron innumerables procesos de control sobre los seres vivos y sobre las expresiones de vida entre ellos (se hace referencia aquí al control sobre los sexos, cuerpos, géneros, sexualidades e identidades sexuales del deseo ${ }^{l}$ ).

Conexo a estos pensamientos, en la perspectiva de Foucault ${ }^{10}$, los dispositivos: a) aparecen de forma heterogénea, dialógica y no jerárquica $\mathrm{y} ; \mathrm{b}$ ) se presentan como un conjunto de discursos y organizaciones arquitectónicas, pues emergen como un tipo de formación que en un determinado contexto histórico respondieron a una necesidad.

\footnotetext{
${ }^{I}$ Para una aproximación mayor a este concepto ver la tesis doctoral de Caravaca-Morera (2016) ${ }^{17}$.
} 


\section{Revista Electrónica Enfermeria Actual en costa Rica}

Por su parte, Lima ${ }^{3}$ en consonancia con Foucault ${ }^{9}$ reconoce que la pluralidad del dispositivo es su característica principal, afirmación que permite declarar que los dispositivos no se componen en sujetos ni en objetos, sino en un régimen de enunciaciones que es necesario definir en función de lo visible y expresable con sus derivaciones, transformaciones y mutaciones. En consecuencia, podría pensarse que estos elementos (no solo descriptivos más prescriptivos) están lejos de configurarse enclaustrados, sino como un conjunto de diferentes linealidades que se articulan y rearticulan constantemente, conservando en su interior la característica de las tensiones y contradicciones que lo circundan.

Como se ha demostrado, por excelencia el dispositivo es contradictorio, pues diseña el juego paradójico establecido entre las personas, su medio y las normativas. En esta perspectiva, es fácil concordar con Agamben ${ }^{16}$,quien en la sustentación de su teoría tanatopolítica, incursiona en el término de dispositivo foucaultiano, pero amplía su análisis histórico al profundizar en los asuntos de orden genealógico, teológico y de desencuentros con la traducción del término griego oikonomia, traducido por algunos filósofos latinos como dispositivo.

Para Agamben, el dispositivo es comprendido como un conjunto de mecanismos políticos contemporáneos que se proliferan para controlar la conducta, los pensamientos y las opiniones de todos los seres humanos ${ }^{16}$; es decir, un dispositivo es cualquier cosa que tenga algún modo o capacidad de capturar, orientar, determinar, interceptar, modelar, controlar y asegurar los gestos, las conductas, opiniones y discursos de los sujetos. De acuerdo con lo expresado, no únicamente las prisiones son dispositivos, puesto que las familias, los manicomios, el panóptico, las escuelas, las confesiones, las fábricas, las disciplinas, las medidas jurídicas, entre otras instituciones sociales, son dispositivos involucrados y moduladores comportamentales del dispositivo trans.

A partir de la noción ampliada sobre los dispositivos de vigilancia y control, las transexualidades son analizadas, escudriñadas e interpretadas en la actualidad a partir de los acontecimientos que las rodean. En este orden de ideas, es necesario reiterar que los dispositivos son históricamente construidos y se singularizan a través de las diferentes experiencias en los espacios que los circunscriben.

A partir de condiciones identitarias fronterizas y a modo de ejemplo, tomando como referente la condición trans, la decisión de colocar la transexualidad en la última versión del DSM-V, como disforia de género, tiene un impacto mundial considerando la potencia de captura y diseminación que este dispositivo posee ${ }^{3}$.

Por su parte, los desdoblamientos de este impacto solo pueden ser percibidos en el ámbito de sus singularidades y de cómo esta vivencia es percibida, vivida y reconocida (o no) en los diferentes contextos sociales y culturales y a través de la interacción con la red de dispositivos clínicos, jurídicos, epistemológicos y mediáticos que la moderan. Lo anterior invita a pensar que el dispositivo de la transexualidad no es más que una invención plástico-tecnológica, que busca estandarizar y uniformar a un conjunto de personas, con una gama de características, dentro de una definición restrictiva y asfixiante.

Afirmar que fue inventado no significa que nunca existió. Al contrario, este presupuesto debería revelar el reconocimiento de cómo, cuándo y de qué maneras esta forma de subjetividad pasó a constituir un elemento tan importante desde el punto de vista discursivo y pragmático para las diversas sociedades. Al respecto, para comprender cómo y en cuáles condiciones el dispositivo de la transexualidad fue concebido, es necesario 


\section{Revista Electrónica Enfermeria Actual en costa Rica}

entenderlo dentro de un macro-contexto de medicalización del cuerpo individual y colectivo que tuvo inicio en las transformaciones de los siglos XVIII y XIX ${ }^{7}$.

En conexión con esos presupuestos, hasta el siglo XVII era posible percibir una relativa tolerancia en relación con las expresiones de género entre los diferentes individuos, pues se vivía bajo los estatutos de una concepción genital isomórfica.

Como ha sido demostrado por la investigación histórica de Lacquer $^{(18)}$, la invención de la diferenciación sexual ilustrada en la cultura occidental por la literatura médica desde la antigüedad hasta el siglo XVII, fue diferente de la actual distinción binaria. Lacquer puntualiza la noción de un cuerpo "unisexuado", es decir, único, en otras palabras, el autor habla de una supuesta convicción de que existía apenas un cuerpo, y por lo menos dos géneros, y de que no existía una noción de hombres y mujeres pertenecientes a sexos inconmensurablemente diferentes y opuestos ${ }^{18,19}$

Este paralelismo de la convención isomórfica se refiere a órganos en el cuerpo femenino que eran vistos como menos desarrollados al compararlos con los masculinos ${ }^{18}$. A partir de esa comprensión se revela la noción poco unívoca que el cuerpo humano diseñaba, ya que la figura femenina era considerada simplemente una más de las graduaciones posibles de esta morfología universal.

Con todo, algunas resistencias, dinámicas y transformaciones en el panorama sociocolectivo hicieron que la complejidad de los discursos pragmáticos del sexo, cuerpo, género y la sexualidad pasara a formar parte del dominio y control absoluto del Estado (ortodoxo), cuyo objetivo primordial estuvo siempre vinculado a la administración de la vida en las esferas públicas, políticas, afectivas y sexuales.

Instituidos a partir del siglo XVIII, los modelos disciplinares sobre los sexos, cuerpos, géneros y sexualidades, se configuraron como una ecuación tensional cuyo objetivo fue organizar, categorizar y universalizar a las personas y sus espacios de enunciación a través de técnicas disciplinares y de clasificación de las conductas en cajas semánticas restrictivas y psiquiátricas.

En ese exacto sentido y de manera complementaria, la génesis del dispositivo (considerado por muchas posturas como patológico) de la transexualidad se inscribió históricamente, dispositivo tal que fue diseñado en la aurora representacional y reproducido en el tejido social por campos del saber como la medicina, específicamente desde las ciencias psi (psiquiatría, psicología y psicoanálisis) ${ }^{5,19}$. En sentido análogo, Foucault afirma que la disciplina desde los diferentes campos de conocimiento, pero principalmente desde la medicina, pasó a configurarse como uno de los principales elementos de funcionamiento en los dispositivos de control panóptico del cuerpo, caracterizado por la inserción de los sujetos en un espacio individualizado, clasificatorio y combinatorio, mediados por la sucesión y confluencia de varios factores estructurales ${ }^{8}$.

Tal como se destacó, medicalizar, patologizar o transformar condiciones multisémicas humanas en "trastornos" plausibles a ser tratados mediante terapias o tratamientos farmacológicos, pasó a ser una de las preocupaciones del siglo XIX, reforzadas en diferentes aspectos durante el siglo XX, principalmente después de la Segunda Guerra Mundial. 


\section{Revista Electrónica Enfermeria Actual en costa Rica}

Esta lógica perversa, favoreció la implantación de estrategias de pensamiento reclusorio que objetiva(ba) la segregación de los cuerpos en una escala dicotómica y binaria, utilizando como brújula justificadora la plasticidad construccionista y materialista de nuestras genitalidades.

Otro elemento irrevocable en esa dinámica creadora del dispositivo de la transexualidad fue la normalización, la cual se tornó en una de las principales herramientas de poder y se constituyó en un campo de fuerzas donde los juegos de verdades ganaron relevancia y los imperativos discursivos y prácticos eran los encargados de la regulación de la vida social.

Aliado con el concepto de disciplina, la normalización de las identidades trans se convirtió en un imperativo modelar en la contemporaneidad, proceso que establece los límites del ser, estar, pensar y sentir, al tiempo que legitima (o excluye) espacios de convivencia con aquellos que se encuentran dentro de la cisnorma.

Luego de partida, esas características limiares se harían presentes en los dominios públicos con el intuito de moldear individuos estandarizados y que cumplieran esos patrones normativos y conductuales igualitarios e impersonales. Efectivamente, es en el ámbito de este continuo que la transexualidad fue significada como patología, consolidándose, como enfermedad per se, durante las décadas de 1940 y 1950 en un movimiento histórico que desde finales del siglo XIX venía produciendo una serie de saberes, discursos y prácticas ${ }^{20}$.

En ese contexto, la creación en 1965 de la primera clínica de identidad sexual del Johns Hopkins Hospital fue fundamental en la discusión y formulación de ideas en torno del concepto de identidad y género. Complementariamente, los trabajos de Robert Stoller (1982), sus tipologías, conducciones clínicas y terapéuticas, fueron claves en la singularización de la transexualidad en cuanto dispositivo patológico ${ }^{3}$.

Se trata de un conjunto de movimientos particulares, institucionales y mecánicos que ayudaron a filtrar las interpretaciones, demandas e intereses de la ciencia médica en relación con el dispositivo trans durante esos años. Sin embargo, nótese la evidencia substantiva de ese discurso viciado por intereses psiquiátricos y frecuentemente raptado por las voluntades y anhelos particularistas de este segmento profesional, que contribuyeron a consolidar la medicalización del dispositivo de la transexualidad con trazos bien delimitados, características diagnósticas específicas y conducciones terapéuticas singulares.

Conforme lo mencionado, y como bien argumenta Foucault, los dispositivos emergen de un determinado momento histórico para responder a una urgencia ${ }^{8}$. En ese sentido, al considerar los límites epistemológicos de las ideas médico-céntricas y antropocéntricas de seres considerados soberanos, tal vez se pueda pensar que la invención del dispositivo trans, en cuanto patología, emergió de la necesidad urgente de clasificar esa condición comportamental y diferenciarla de la homosexualidad, travestilidad y de las condiciones intersexuales;es decir, ante este panorama, el discurso sociológico contemporáneo consideró necesario definir y caracterizar al fenómeno trans para separarlo de otros conjuntos de prácticas vivenciales y "disturbios psiquiátricos".

Podría mencionarse que las relativamente recientes elaboraciones biopolíticas en torno de la idea del dispositivo medicalizado de la transexualidad ambicionan contemplar tales críticas dirigidas a la normalización y estandarización de los cuerpos considerados sáceres.

Pensar en biopolítica lleva a la comprensión de que este proceso no se constituye en otro (o un nuevo) régimen de poder, sino en un acoplamiento del poder sobre las sociedades disciplinares, cuyas características 


\section{Revista Electrónica Enfermeria Actual en costa Rica}

incluyen, entre otras, la disciplinarización de los cuerpos individuales y colectivos, así como su adiestramiento a partir de una norma establecida.

Pese a ello, lo que comienza a cambiar a partir del siglo XVII y, principalmente a partir del siglo XVIII, es la invención del concepto de población y de cómo esta, con todo su aparato logístico, pasó a ser el espacio sobre el cual la relación saber/poder e incitación/control actuarían. En ese sentido, la propia definición de "norma" y, consecuentemente, de lo "normal" (en el sentido goffminano) y de lo "enfermo" (en el sentido nosológico) sufrirán alteraciones considerables. En uno de sus ensayos, Focault explicó las diferencias entre las sociedades disciplinares y la biopolítica, argumentando que en los regímenes disciplinares se parte siempre de una norma y que era el adiestramiento efectuado por la norma lo que posibilitaría a posteriori la distinción entre lo normal y lo abyecto $^{22}$.

Complementariamente, menciona que en las ontologías humanas (aquí en cuestión las identidades trans) es posible identificar las diferentes curvas de normalidad, y que la operación de la normalización va a consistir en hacer que esas diferentes distribuciones de normalidad funcionen en relación con las otras. Para el filósofo francés, son esas distribuciones las que funcionarán como norma. Lo normal es anterior y la norma se desprende de ello; en palabras simples: a partir del estudio de las normalidades, la norma se fija y desempeña su papel operatorio: operaciones de normalización ${ }^{9}$.

Esta lógica transformó significativamente la relación con las normas, las cuales no constituían más el marco límite que separaba la normalidad (razón) de la anormalidad (locura). Ahora, las interacciones sociocolectivas pasarían a componer tonalidades de normalización, donde nadie y nada podría escapar.

Basta recordar el cuadro clínico creado por Harry Benjamin para explicar el llamado "proceso transexual", que decretaba transexualidades en escalas que iban del 1 al 6 , siendo el tipo 1 considerado como pseudotravesti y el tipo 6, como la persona transexual "verdadera",22.

Vislumbrada como singular en relación con otros dispositivos políticos, la transexualidad se sitúa como uno de los fenómenos más expresivos en la biopolítica contemporánea, puesto que en el ámbito de los propios dispositivos -espacios de poder y control- operan procesos de sumisión que refuerzan códigos culturales, discursos y prácticas cisheteronormativas.

Partiendo de esta realidad, para la biopolítica contemporánea es necesario transportar la potencia de los cuerpos y sus singularidades. Sobre esta posibilidad, reside la apuesta de potenciar modos de vivir (se), no fuera ni detrás de los dispositivos de control, sino dentro de sus ámbitos, cotidianidad y actualidad.

Mucho ya se ha debatido sobe la caracterización de la postura patologizante que medicaliza al dispositivo trans, lo cual reduce y restringe las singularidades, pluralidades y posibilidades de modos de existencia y resistencia de esta población. Constituye parte de la comprensión integral de este dispositivo, el escuchar atentamente al clamor retumbante de las voces consideradas como ininteligibles o subalternas, que protagonizan la historia de la transexualidad como uno de los fenómenos más singulares en el ámbito de las resignificaciones del género en la contemporaneidad. 


\section{Revista Electrónica Enfermeria Actual en costa Rica}

A partir de esta realidad se revela la "necesidad" de considerar en el debate de salud actual lo que se encuentra más allá de los discursos y prácticas circunscritas a los espacios médicos (hospitales y servicios), o a lo cotidiano de las experiencias y vivencias de las transexualidades.

\section{CONCLUSIONES}

La transexualidad es uno de los temas privilegiados en el debate actual sobre los sexos, cuerpos, géneros, sexualidades e identidades sexuales del deseo. La comprensión de este proceso, así como de sus transformaciones, depende del análisis y reflexión crítica de la transexualidad como dispositivo.

El surgimiento de la sociedad disciplinar mencionada en los postulados foucaultianos, fue una condición sine qua non presente en la (re)configuración de las relaciones entre saber-poder y formas de control del cuerpo, tanto individual (anátomo-político) como colectivo. En lo interior de estas relaciones, los sexos, cuerpos, géneros y sexualidades - su polisemia y polimórfica semántica - fueron capturadas por el modelo biopolítico, transformándolas en un espacio de control, disciplinarización, producción de verdades e invenciones, constituidos a partir de la integración de diferentes saberes (principalmente desde las ciencias psi) diseminados por el tejido social (micropoder).

Precisamente, a partir de las relaciones establecidas entre los diferentes dominios de conocimiento, principalmente entre la Medicina (desde la Psiquiatría), el Derecho, la Psicología y la Sociología, fue que la transexualidad se transformó en un dispositivo anclado en el ámbito de los trastornos de salud mental.

En ese exacto sentido, la construcción de la norma génerico-sexual se constituyó en un movimiento que, a partir de finales del siglo XIX e inicio del siglo XX, comenzó a (re)producir discursos mecanicistas sobre los sujetos, sexos, cuerpos, géneros y sexualidades.

En este contexto, las discusiones se forjaron en oposición a las ideas de la homosexualidad, travestilidad y, principalmente, de la intersexualidad. Esta separación y singularización se dio a partir de una postura nosológica y nosográfica propia que delimitó las fronteras, las características y las condiciones terapéuticas.

La norma - la idea de la transexualidad como trastorno de identidad de género - se implantó de forma imperativa, caracterizando y determinando los comportamientos que una persona autoidentificada como trans debía performatizar. En conjunto, esos acontecimientos, compuestos por las producciones discursivas, postulados, tesis científicas, investigaciones, experiencias e instituciones sociales, componen lo que hoy denominamos como dispositivo trans.

A través de este análisis fue posible percibir cómo se da la (re)interacción de las personas trans con la norma psiquiátrica, entendiendo este movimiento como un proceso marcado significativamente por las relaciones de poder y comprendiendo que donde hay poder, hay posibilidades de resistencia y subversiones de la norma.

Finalmente, a través de este recorrido sucinto es posible (re)pensar el proceso de patologización del dispositivo trans, entendiéndolo como una posibilidad de resistencia y contestación al imperativo cisheteronormativo y a la medicalización de condiciones diversas y por veces inefables. 


\section{Revista Electrónica Enfermeria Actual en costa Rica}

\section{BIBLIOGRAFÍA}

1. Preciado, B. Manifiesto contra-sexual: prácticas subversivas de identidad sexual. Madrid: Pensamiento Opera Prima; 2002.

2. Butler, J. Desdiagnosticando o gênero. Physis Revista de Saúde Coletiva. 2009; 19(1): 95-126.

3. Lima-Santos, M.F. Corpos, Gêneros, Sexualidades: Políticas de Subjetivação. Porto Alegre: Rede UNIDA; 2014.

4. Bento, B. "Transexuais, corpos e próteses". Labrys: Estudos Feministas. 2013; 14(3): 1-10.

5. Conrad, P. The Medicalization of Society: On the Transformation of Human Conditions into Treatable Disorders. Baltimore: Johns Hopkins University Press; 2007.

6. Butler, J. Undoing gender. Nueva York: Routledge; 2004.

7. Foucault, M. História da sexualidade: a vontade de saber. Rio de Janeiro: Paz e Terra; 2014.

8. Foucault, M. Microfísica do poder. $28^{\mathrm{a}}$ ed. Rio de Janeiro: Record; 2013.

9. Foucault, M. O Nascimento da Biopolítica. São Paulo: Martins Fontes; 2007.

10. Foucault, M. O Sujeito e o Poder. In Dreyfus, H. L e Rabinow, P. Michel Foucault - uma trajetória filosófica. Rio de Janeiro: Forense Universitária; 2012.

11. Deleuze, G. ¿Qué es un dispositivo? En Michel Foucault, filósofo. Barcelona: Gedisa; 1990.

12. Foucault, M. Segurança, Território e População. São Paulo: Martins Fontes; 2008.

13. Foucault, M. Vigiar e Punir. Petrópolis: Vozes; 2015.

14. American Psychiatric Association. DSM-4-TR: Manual diagnóstico e estatístico de transtornos mentais (ed. rev.). Porto Alegre, RS: Artes Médicas; 2003.

15. American Psychiatric Association. DSM 5 Diagnostic and Statistical Manual of Mental Disorders: Portland: APA; 2013.

16. Agamben, G. O que é um dispositivo? Santa Catarina: Outra travessia; 2005.

17. Caravaca-Morera, JA. Trans: Histórias de vida e Representações sociais do Sexo, Corpo, Gênero e Sexualidade entre pessoas transexuais do Brasil, Canadá e Costa Rica. Tese de Doutorado: Universidade Federal de Santa Catarina; 2016.

18. Laquer, T. Making Sex: Body and Gender from the Greeks to Freud. Cambridge (Massachusetts): Harvard University Press; 1990.

19. Cauldwell, DO. Psychopathia transexualis. International Journal of Trangenderism. 2001; 5(2): 171-191.

20. Ávila, S. El género desordenado: críticas en torno a la patologización de la transexualidad. Cadernos Pagu. 2012; 38(1): 441-451.

21. Foucault, M. Em defesa da sociedade. São Paulo: Martins Fontes; 2000.

22. Benjamin, H. The transsexual phenomenon. New York: Julian; 1966. 